Supporting Information

\title{
Aromatic Foldamers with Iminodicarbonyl Linkers: Their Structures and Optical Properties
}

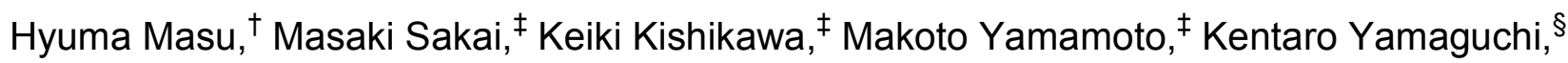 \\ and Shigeo Kohmoto*, $\dagger$
}

Graduate School of Science and Technology and Department of Chemistry and Biotechnology, Faculty of Engineering,, Chiba University, 1-33, Yayoi-cho, Inage-ku, Chiba 263-8522, Japan

Department of Pharmaceutics, Faculty of Pharmaceutical Science, Tokushima Bunri University Shido, Sanuki-city, Kagawa 769-2193 Japan

\section{TABLE OF CONTENTS}

- General Experimental Section $\quad$ S2

- ${ }^{1} \mathrm{H}$ NMR Spectrum of $\mathbf{2 c}$ S9

- ${ }^{1}$ H NMR Spectrum of $\mathbf{5 b} \quad \mathrm{S} 10$

- ${ }^{1} \mathrm{H}$ NMR Spectrum of $\mathbf{5 c} \quad \mathrm{S} 11$

- ORTEP diagram of 3a $\quad \mathrm{S} 12$

- ORTEP diagram of $\mathbf{3 b} \quad \mathrm{S} 13$

- ORTEP diagram of 3c $\quad \mathrm{S} 14$

- ORTEP diagram of 3d $\quad$ S15

- ORTEP diagram of 3e $\quad$ S16 
General Information. Common commercial reagents were used as received from the commercial suppliers without further purification. IR spectra were recorded as $\mathrm{KBr}$ pellets. Intensities of peaks are reported as: s (strong), $\mathrm{m}$ (middle), w (weak), br (broad). UV-Vis absorption spectra are presented as $\lambda_{\max }$ and $\varepsilon_{\max } .{ }^{1} \mathrm{H}$ and ${ }^{13} \mathrm{C}$ NMR spectra were obtained in $\mathrm{CDCl}_{3}$ solutions. Chemical shifts are reported in delta $(\delta)$ units, parts per million (ppm) relative to the TMS as internal standard. Coupling constants $J$ are in Hz. Low and high resolution mass spectra were obtained in the FAB mode, as specified. Several crystalline compounds included solvent or water molecules. This was taken into account in calculation of elemental analysis. Unless otherwise noted, each spectrum was measured at ambient temperature.

\section{Preparation of Compounds.}

Benzylamine $(1.12 \mathrm{~g}, 10.2 \mathrm{mmol})$ and triethylamine $(1.80 \mathrm{ml}, 13.0 \mathrm{mmol})$ in toluene $(50 \mathrm{ml})$ were added to the toluene solution of 1,4-naphthalenedicarbonylchloride prepared from 1,4-naphthalenedicarboxylic acid $(1.01 \mathrm{~g}, 4.67 \mathrm{mmol})$ with thionylchloride and the resulting solution was refluxed for $8 \mathrm{~h}$. After cooling to room temperature, the precipitate was filtrated in vacuum and washed with chloroform to give $1 \mathrm{a}$ as a white powder. No further purification was necessary. Yield $1.82 \mathrm{~g}(99 \%)$; m.p. 238-239 ${ }^{\circ} \mathrm{C}$. IR (KBr) 3448 (br), 1700 (m),1660 (s) cm ${ }^{-1}$; UV-vis $\left(\mathrm{CHCl}_{3}\right) 295$ (7800) nm; ${ }^{1} \mathrm{H}$ NMR $\left(\mathrm{CDCl}_{3}, 500\right.$ MHz) $\delta 8.20(\mathrm{dd}, J=6.5,3.3,2 \mathrm{H}), 7.56(\mathrm{dd}, J=6.5,3.4,2 \mathrm{H}), 7.53(\mathrm{~s}, 2 \mathrm{H}), 7.43-7.36(\mathrm{~m}, 10 \mathrm{H}), 7.31(\mathrm{tt}$, $J=7.2,1.7,2 \mathrm{H}), 4.69$ (s, 4H); ${ }^{13} \mathrm{C} \mathrm{NMR}\left(\mathrm{CDCl}_{3}, 125 \mathrm{MHz}\right) \delta 169.8(\mathrm{~s}), 137.8(\mathrm{~s}), 136.2(\mathrm{~s}), 130.0(\mathrm{~s})$, 128.5 (d), 127.6 (d), 127.3 (d), 127.2 (d), 125.3 (d), 123.5 (d), 43.7 (t); MS (FAB) m/z 395 [MH $\left.{ }^{+}\right]$. Anal. Calcd. for $\mathrm{C}_{26} \mathrm{H}_{22} \mathrm{~N}_{2} \mathrm{O}_{2} \cdot 2 / 3 \mathrm{H}_{2} \mathrm{O}: \mathrm{C}, 76.83 ; \mathrm{H}, 5.79 ; \mathrm{N}, 6.89$. Found: C, 76.70; H, 5.68; N, 7.05.

In a similar way as for $\mathbf{1 a}$, amides $\mathbf{1 b}, \mathbf{1 c}, \mathbf{1 d}$, and $\mathbf{1 e}$ were prepared in $76,72,85$, and $60 \%$ yields, respectively.

Naphthalene-1,4-dicarboxylic acid bis-[((1S)-phenylethyl)amide] 1b. White powder, m.p. 246-248 ${ }^{\circ} \mathrm{C}$. IR (KBr) 3422 (br), 1630 (s),1530 (m) cm ${ }^{-1}$; UV-vis $\left(\mathrm{CH}_{3} \mathrm{CN}\right) 226$ (65000), 289 (11000) nm; ${ }^{1} \mathrm{H}$ NMR $\left(\mathrm{CDCl}_{3}, 500 \mathrm{MHz}\right) \delta 8.00(\mathrm{~s}, 2 \mathrm{H}), 7.43-7.34(\mathrm{~m}, 12 \mathrm{H}), 7.29(\mathrm{t}, J=7.2,2 \mathrm{H}), 5.38(\mathrm{q}, J=6.4,2 \mathrm{H}), 3.69$ (br, 2H), $1.63(\mathrm{~d}, J=6.8,6 \mathrm{H}) ;{ }^{13} \mathrm{C} \mathrm{NMR}\left(\mathrm{CDCl}_{3}, 125 \mathrm{MHz}\right) \delta 143.1(\mathrm{~s}), 136.1(\mathrm{~s}), 129.8$ (s), $128.5(\mathrm{~d})$, 127.2 (d), 127.0 (d), 126.0 (d), 125.2 (d), 123.4 (d), 49.2 (d), 21.6 (q); MS (FAB) m/z 422 [M ]. Anal. Calcd. for $\mathrm{C}_{28} \mathrm{H}_{26} \mathrm{~N}_{2} \mathrm{O}_{2} \cdot 1 / 2 \mathrm{H}_{2} \mathrm{O}$ : C, 77.93; H, 6.31; N, 6.49. Found: C, 77.94; H, 6.11; N, 6.47. 
Naphthalene-1,4-dicarboxylicacid bis-[((1S)-(1-naphthyl)ethyl)amide] 1c. White powder, m.p. $158{ }^{\circ} \mathrm{C}$. IR (KBr) 3272 (br), 3052 (w), 1641 (s), 1524 (s), (w) cm ${ }^{-1}$; UV-vis $\left(\mathrm{CH}_{3} \mathrm{CN}\right) 274$ (16000), 283 (21000), $293(19000) \mathrm{nm} ;{ }^{1} \mathrm{H} \mathrm{NMR}\left(\mathrm{CDCl}_{3}, 500 \mathrm{MHz}\right) \delta 8.25(\mathrm{~d}, J=8.6,2 \mathrm{H}), 8.12(\mathrm{~m}, 2 \mathrm{H}), 7.87(\mathrm{~d}, J=7.7,2 \mathrm{H})$, $7.79(\mathrm{~d}, J=8.3), 7.57-7.48(\mathrm{~m}, 6 \mathrm{H}), 7.40(\mathrm{t}, J=7.6,2 \mathrm{H}), 7.36(\mathrm{~m}, 2 \mathrm{H}), 7.23(\mathrm{~s}, 2 \mathrm{H}), 6.46(\mathrm{~d}, J=8.2$, 2H), 6.18 (quint, $J=7.2,2 \mathrm{H}), 1.82(\mathrm{~d}, J=6.7,6 \mathrm{H}) ;{ }^{13} \mathrm{C} \mathrm{NMR}\left(\mathrm{CDCl}_{3}, 125 \mathrm{MHz}\right) \delta 168.0(\mathrm{~s}), 138.1(\mathrm{~s})$, 136.6 (s), 134.1 (s), 131.3 (s), 130.3 (s), 129.0 (d), 128.6 (d), 127.5 (d), 126.8 (d), 126.1 (d), 125.6 (d), 125.3 (d), 123.52 (d), 123.48 (d), 122.8 (d), 45.4 (d), 20.8 (q); MS (FAB) $m / z 522$ [M ${ }^{+}$. Anal. Calcd. for $\mathrm{C}_{36} \mathrm{H}_{30} \mathrm{~N}_{2} \mathrm{O}_{2}: \mathrm{C}, 82.73 ; \mathrm{H}, 5.79 ; \mathrm{N}, 5.36$. Found: C, 82.33; H, 5.78; N, 5.30.

Naphthalene-1,4-dicarboxylic acid bis-[(2-tert-butyl-phenyl)-amide] 1d. White powder, m.p. 259-261 ${ }^{\circ} \mathrm{C}$. IR (KBr) 3234 (br), 2966 (w), 1646 (s), 1513 (s) cm ${ }^{-1}$; UV-vis $\left(\mathrm{CH}_{3} \mathrm{CN}\right) 255$ (5900), 292 (10000) nm; ${ }^{1} \mathrm{H}$ NMR $\left(\mathrm{CDCl}_{3}, 500 \mathrm{MHz}\right) \delta 8.46$ (br, 2H), 7.95 (br, 2H), 7.82 (br, 2H), 7.78 (br, 2H), 7.67 (br,2H), 7.48 (br, 2H), 7.37 (br, 2H), 7.26 (br, 2H), 1.43 (m, 18H); ${ }^{13} \mathrm{C}$ NMR $\left(\mathrm{CDCl}_{3}, 125 \mathrm{MHz}\right) \delta 167.2$ (s), 142.5 (s), 137.5 (s), 135.0 (s), 130.6 (s), 128.1 (d), 127.5 (d), 127.1 (d), 126.9 (d), 126.7 (d), 125.8 (d), 123.4 (d), 34.7 (s), 30.8 (q); MS (FAB) $m / z 478\left[\mathrm{M}^{+}\right]$.

Naphthalene-1,4-dicarboxylic acid bis-octylamide 1e. White powder, m.p. 169-173 ${ }^{\circ} \mathrm{C}$. IR (KBr) 3299 (br), 2925 (m), 2854 (m), 1634 (s), 1534 (m) cm ${ }^{-1}$; UV-vis $\left(\mathrm{CH}_{3} \mathrm{CN}\right) 226$ (5200), 289 (1000) nm; ${ }^{1} \mathrm{H}$ $\operatorname{NMR}\left(\mathrm{CDCl}_{3}, 500 \mathrm{MHz}\right) \delta 8.17(\mathrm{~m}, 2 \mathrm{H}), 7.51(\mathrm{~d}, J=6.4,1 \mathrm{H}), 7.51(\mathrm{~d}, J=6.7,1 \mathrm{H}), 7.35(\mathrm{~s}, 2 \mathrm{H}), 6.33$ (br, 2H), 3.51 (q, $J=6.7,4 \mathrm{H}), 1.67$ (quint, $J=7.3,4 \mathrm{H}), 1.44-1.30(\mathrm{~m}, 20 \mathrm{H}), 0.90(\mathrm{t}, J=7.0,6 \mathrm{H}) ;{ }^{13} \mathrm{C}$ $\operatorname{NMR}\left(\mathrm{CDCl}_{3}, 125 \mathrm{MHz}\right) \delta 169.1$ (s), 136.7 (s), 130.2 (s), 127.3 (d), 125.6 (d), 123.6 (d), 40.2 (t), 31.8 $(\mathrm{t}), 29.6(\mathrm{t}), 29.29(\mathrm{t}), 29.26(\mathrm{t}), 27.1(\mathrm{t}), 22.7(\mathrm{t}), 14.1(\mathrm{q})$; MS (FAB) $m / z 438\left[\mathrm{M}^{+}\right]$.

4-[Benzyl-(naphthalene-1-carbonyl)-aminocarbonyl]-naphthalene-1-carboxylic acid benzylamide $2 \mathbf{2 a}$. Naphthalene-1-carbonylchloride $(0.39 \mathrm{ml}, 2.59 \mathrm{mmol})$ and DMAP $(156 \mathrm{mg}, 2.59 \mathrm{mmol})$ was added to $1 \mathbf{a}(1.02 \mathrm{~g}, 2.59 \mathrm{mmol})$ in pyridine $(10 \mathrm{ml})$ and toluene $(5 \mathrm{ml})$. The resulting solution was refluxed for 10 h. After cooling to room temperature, the solution was washed with diluted $\mathrm{HCl}$. The organic layer was dried over $\mathrm{MgSO}_{4}$. After evaporation of solvent, the residues were purified by silica gel column chromatography with $n$-hexane / ethyl acetate $(3: 1)$ as eluent. Recrystallization from chloroform gave 2a as white crystals. Yield 465 mg (33 \%); m.p. 122-125 ${ }^{\circ} \mathrm{C}$. IR (KBr) 3031 (br), 1705 (m), 1654 (s), 1590 (m), 1534 (s) cm ${ }^{-1}$; UV-vis $\left(\mathrm{CHCl}_{3}\right) 301$ (12000), 331 (6200) nm; ${ }^{1} \mathrm{H} \mathrm{NMR}\left(\mathrm{CDCl}_{3}, 500 \mathrm{MHz}\right) \delta$ 7.81-7.77 (m, 2H), $7.74(\mathrm{~d}, J=7.0,2 \mathrm{H}), 7.45-7.42(\mathrm{~m}, 5 \mathrm{H}), 7.38-7.34(\mathrm{~m}, 5 \mathrm{H}), 7.31(\mathrm{~d}, J=7.3,2 \mathrm{H})$, $7.19(\mathrm{t}, J=8.3,1 \mathrm{H}), 7.12(\mathrm{~d}, J=8.3,1 \mathrm{H}), 7.04(\mathrm{~d}, J=8.3,1 \mathrm{H}), 7.01(\mathrm{t}, J=7.0,2 \mathrm{H}), 6.79(\mathrm{t}, J=7.0,1 \mathrm{H})$, $6.66(\mathrm{~d}, J=7.4,1 \mathrm{H}), 6.59(\mathrm{br}, 1 \mathrm{H}), 5.42(\mathrm{~s}, 2 \mathrm{H}), 5.16(\mathrm{br}, 1 \mathrm{H}), 4.48(\mathrm{~d}, J=5.5,2 \mathrm{H}) ;{ }^{13} \mathrm{C} \mathrm{NMR}\left(\mathrm{CDCl}_{3}\right.$, $125 \mathrm{MHz}$ ) $\delta 172.9$ (s), 172.3 (s), 167.9 (s), 137.6 (s), 137.2 (s), 136.04 (s), 136.03 (s), 134.0 (s), 132.4 
(s), 129.9 (d), 129.5 (d), 129.0 (d), 128.81 (d), 128.78 (d), 128.7 (d), 128.0 (d), 127.88 (d), 127.87 (d), 127.4 (d), 126.4 (d), 125.94 (d), 125.91 (d), 125.2 (d), 127.4 (d), 125.1 (d), 124.7 (d), 123.9 (d), 122.3 (d), 48.5 (d), 43.9 (t); MS (FAB) $m / z 549\left[\mathrm{M}^{+}\right]$. Anal. Calcd. for $\mathrm{C}_{37} \mathrm{H}_{28} \mathrm{~N}_{2} \mathrm{O}_{3} \cdot 1 / 2 \mathrm{H}_{2} \mathrm{O}: \mathrm{C}, 80.12 ; \mathrm{H}$, 5.21; N, 5.05. Found: C, 80.18; H, 5.17; N, 4.93 .

In a similar way as for $\mathbf{2 a}, \mathbf{2} \mathbf{b}$ and $\mathbf{2} \mathbf{c}$ were prepared in 20 , and $54 \%$ yields, respectively.

4-(Naphthalene-1-carbonyl)-[(1S)-phenylethyl-aminocarbonyl]-naphthalene-1-carboxylicacid （(1S)phenylethyl)amide 2b. White powder, m.p. 175-176 ${ }^{\circ} \mathrm{C}$. IR (KBr) 3060 (br), 1702 (s), 1657 (s), 1497 (m) $\mathrm{cm}^{-1}$; UV-vis $\left(\mathrm{CH}_{3} \mathrm{CN}\right)$ 250(23000), 300 (11000), 335 (6000) nm; ${ }^{1} \mathrm{H}$ NMR $\left(\mathrm{CDCl}_{3}, 500 \mathrm{MHz}\right) \delta$ $7.89(\mathrm{~d}, J=7.1,1 \mathrm{H}), 7.82(\mathrm{br}, 1 \mathrm{H}), 7.79(\mathrm{~d}, J=7.6,2 \mathrm{H}), 7.68(\mathrm{~m}, 1 \mathrm{H}), 7.47-7.42(\mathrm{~m}, 4 \mathrm{H}), 7.37-7.30(\mathrm{~m}$, $6 \mathrm{H}), 7.22-7.17(\mathrm{~m}, 3 \mathrm{H}), 7.13(\mathrm{~d}, J=7.1,1 \mathrm{H}), 7.08(\mathrm{~d}, J=8.3,1 \mathrm{H}), 6.89(\mathrm{t}, J=7.3,1 \mathrm{H}), 6.75-6.70(\mathrm{~m}$, 2H), $6.36(\mathrm{q}, J=7.2,1 \mathrm{H}), 5.21(5, J=7.0,1 \mathrm{H}), 5.15(\mathrm{~m}, 1 \mathrm{H}), 2.15(\mathrm{~d}, J=6.3,3 \mathrm{H}), 1.58(\mathrm{~d}, J=6.6$, 3H); ${ }^{13} \mathrm{C} \mathrm{NMR}\left(\mathrm{CDCl}_{3}, 125 \mathrm{MHz}\right) \delta 172.9$ (s), 172.1 (s), 167.3 (s), 142.6 (s), 136.7 (s), 136.4 (s), 134.8 (s), 132.6 (s), 130.2 (d), 129.1 (s), 129.0 (s), 128.8 (d), 128.4 (d), 128.1 (d), 127.8 (d), 127.7 (d), 127.4 (d), 127.2 (d), 126.3 (d), 126.2 (d), 125.5 (d), 125.2 (d), 124.84 (d), 124.78 (d), 124.2 (d), 122.4 (d), 55.1 (d), 49.0 (d), 21.3 (q), 17.5 (q); MS (FAB) $m / z 577\left[\mathrm{MH}^{+}\right]$. Anal. Calcd. for $\mathrm{C}_{39} \mathrm{H}_{32} \mathrm{~N}_{2} \mathrm{O}_{3} \cdot 1 / 2 \mathrm{H}_{2} \mathrm{O}: \mathrm{C}$, 79.98; H, 5.68; N, 4.78. Found: C, 80.17; H, 5.63; N, 4.68.

4-(Naphthalene-1-carbonyl)-[(1S)-(1-naphthyl)ethyl]-aminocarbonyl]-naphthalene-1-carboxylic acid [(1S)-(1-naphthyl)ethyl]amide 2c. m.p. 115-120 ${ }^{\circ} \mathrm{C}$. IR (KBr) 3052 (w), 1702 (m), 1654 (s), 1513 (m) $\mathrm{cm}^{-1}$; UV-vis $\left(\mathrm{CH}_{3} \mathrm{CN}\right) 226$ (110000), 272 (15000), 294 (17000) nm; ${ }^{1} \mathrm{H}$ NMR $\left(\mathrm{CDCl}_{3}, 500 \mathrm{MHz}\right) \delta$ $8.27(\mathrm{~d}, J=8.6,1 \mathrm{H}), 8.16(\mathrm{~d}, J=8.6,1 \mathrm{H}), 8.08-8.05(\mathrm{~m}, 2 \mathrm{H}), 7.97-7.79(\mathrm{~m}, 6 \mathrm{H}), 7.61-7.47(\mathrm{~m}, 9 \mathrm{H})$, $7.41(\mathrm{t}, J=7.6,1 \mathrm{H}), 7.28-7.24(\mathrm{~m}, 2 \mathrm{H}), 7.06-6.77(\mathrm{~m}, 4 \mathrm{H}), 6.47$ (br, 2H), 6.21 (quint, $J=7.0,1 \mathrm{H}), 5.93$ (quint, $J=7.3,1 \mathrm{H}), 4.97(\mathrm{br}, 1 \mathrm{H}), 1.82(\mathrm{~d}, J=6.7,3 \mathrm{H}), 1.74(\mathrm{~d}, J=6.7,3 \mathrm{H}) ;{ }^{13} \mathrm{C} \mathrm{NMR}\left(\mathrm{CDCl}_{3}, 125\right.$ MHz) $\delta 167.1$ (s), 166.9 (s), 139.1 (s), 137.6 (s), 137.4 (s), 136.2 (s), 134.00 (s), 133.95 (s), 133.8 (s), 132.1 (s), 131.3 (d), 131.1 (s), 129.9 (d), 129.6 (s), 129.2 (d), 129.0 (d), 128.90 (d), 128.87 (d), 128.85 (d), 128.6 (d), 128.4 (d), 127.3 (d), 127.2 (d), 126.7 (d), 126.1 (d), 126.04 (d), 125.98 (d), 125.8 (s), 125.6 (d), 125.2 (d), 125.1 (d), 124.0 (d), 123.4 (d), 123.3 (d), 123.2 (d), 123.1 (s), 122.8 (d), 122.7 (d), 122.2 (d), 45.2 (d), 45.0 (d), 20.5 (q), 19.8 (q); HRMS (FAB), calcd for $\mathrm{C}_{47} \mathrm{H}_{36} \mathrm{~N}_{2} \mathrm{O}_{3}\left(\mathrm{M}^{+}\right)$676.2726, found 676.2672 .

$\mathrm{N}$-(naphthylcarbonyl) $\{4-[\mathrm{N}-($ naphthylcarbonyl)-N-benzylcarbamoyl] naphthyl $\}-\mathrm{N}-$ benzylcarboxamide 3a. Naphthalene-1-carbonylchloride $(0.56 \mathrm{ml}, 3.72 \mathrm{mmol})$ and DMAP $(454 \mathrm{mg}, 3.72 \mathrm{mmol})$ were added to a solution of $1 \mathrm{a}(665 \mathrm{mg}, 1.69 \mathrm{mmol})$ in pyridine $(7 \mathrm{ml})$ and toluene $(7 \mathrm{ml})$. The resulting mixture was 
refluxed for $5 \mathrm{~h}$. After cooling to room temperature, the solution was washed with aqueous $1 \mathrm{~N} \mathrm{HCl}$ solution. The organic layer was dried over $\mathrm{MgSO}_{4}$. After filtration, solvent was evaporated and the residue was purified by silica gel column chromatography with $n$-hexane / ethylacetate $(3: 1)$ as eluent. Recrystallization from chloroform gave 3a $(688 \mathrm{mg}, 58 \%)$ as colorless crystals. m.p. $107-113{ }^{\circ} \mathrm{C}$. IR (KBr) 3061 (br), 1701 (m), 1660 (s), 1509 (w) cm-1; UV-vis $\left(\mathrm{CHCl}_{3}\right) 305$ (9300), 344 (6000) nm; ${ }^{1} \mathrm{H}$ $\operatorname{NMR}\left(\mathrm{CDCl}_{3}, 500 \mathrm{MHz}\right) \delta 7.60(\mathrm{dd}, J=8.0,1.5,4 \mathrm{H}), 7.52(\mathrm{~d}, J=8.0,2 \mathrm{H}), 7.47(\mathrm{t}, J=7.3,4 \mathrm{H}), 7.41$ $(\mathrm{tt}, J=6.3,1.3,2 \mathrm{H}), 7.37(\mathrm{br}, 2 \mathrm{H}), 7.28(\mathrm{dd}, J=8.0,1.5,2 \mathrm{H}), 7.13(\mathrm{~m}, 2 \mathrm{H}), 7.09(\mathrm{td}, J=7.5,1.5,2 \mathrm{H})$, $7.06(\mathrm{td}, J=6.6,1.1,2 \mathrm{H}), 6.97(\mathrm{~d}, J=8.2,2 \mathrm{H}), 6.82(\mathrm{dd}, J=7.0,1.0,2 \mathrm{H}), 6.43(\mathrm{t}, J=7.5,2 \mathrm{H}), 6.20(\mathrm{~s}$, 2H), $5.24(\mathrm{~s}, 4 \mathrm{H}) ;{ }^{13} \mathrm{C} \mathrm{NMR}\left(\mathrm{CDCl}_{3}, 125 \mathrm{MHz}\right) \delta 172.8$ (s), 171.2 (s), 137.3 (s), 135.1 (s), 133.5 (s), 132.3 (s), 130.1 (s), 129.4 (d), 128.6 (d), 128.6 (s), 128.2 (d), 128.1 (s), 127.9 (d), 126.8 (d), 126.2 (d), 125.7 (d), 124.5 (d), 124.1 (d), 123.3 (d), 122.9 (d), 48.3 (t); MS (FAB) $m / z 703\left[\mathrm{MH}^{+}\right]$. Anal. Calcd. for $\mathrm{C}_{48} \mathrm{H}_{34} \mathrm{~N}_{2} \mathrm{O}_{4} \cdot 2 / 3 \mathrm{H}_{2} \mathrm{O}: \mathrm{C}, 80.65 ; \mathrm{H}, 4.98 ; \mathrm{N}, 3.92$. Found: $\mathrm{C}, 80.53 ; \mathrm{H}, 4.83 ; \mathrm{N}, 3.91$.

In a similar manner as for $\mathbf{3 a}, \mathbf{3 b}, \mathbf{3 c}, \mathbf{3 d}$, and $\mathbf{3 e}$ were prepared in $46,35,11$, and $20 \%$ yields, respectively.

$\mathrm{N}-($ naphthylcarbonyl) \{4-[N-(naphthylcarbonyl)-N-((IS)-phenylethyl)carbamoyl]naphthyl $\}-\mathrm{N}-((1 S)-$ phenylethyl)carboxamide 3b. Colorless crystals, m.p. 109-111 ${ }^{\circ} \mathrm{C}$. IR (KBr) 3057 (w), 1700 (m), 1658 (s), $1313(\mathrm{~m}) \mathrm{cm}^{-1}$; UV-vis $\left(\mathrm{CH}_{3} \mathrm{CN}\right) 260$ (22000), 303 (11000), 350 (6800) nm; ${ }^{1} \mathrm{H}$ NMR (CDCl, 500 MHz) $\delta 7.68(\mathrm{~d}, J=7.7,4 \mathrm{H}), 7.59(\mathrm{~d}, J=8.0,2 \mathrm{H}), 7.50(\mathrm{t}, J=7.6,4 \mathrm{H}), 7.41(\mathrm{t}, J=7.6,2 \mathrm{H}), 7.38(\mathrm{br}$, 2H), 7.22 (d, $J=7.1,2 \mathrm{H}), 7.08-7.05(\mathrm{~m}, 4 \mathrm{H}), 7.07$ (m, 2H), $6.94(\mathrm{~d}, J=6.7,4 \mathrm{H}), 6.49$ (t, $J=7.5,2 \mathrm{H})$, 6.41 (s, 2H), 6.16 (q, 2H), 2.02 (br, 6H); ${ }^{13} \mathrm{C} \mathrm{NMR}\left(\mathrm{CDCl}_{3}, 125 \mathrm{MHz}\right) \delta 172.7$ (s), 171.0 (s), 135.9 (s), 134.3 (s), 132.3 (s), 130.2 (d), 128.4 (s), 128.3 (d), 128.1 (d), 128.0 (d), 127.9 (s), 127.6 (d), 126.7 (d), 126.3 (d), 125.8 (d), 124.6 (d), 124.2 (d), 123.53 (d), 123.50 (s), 123.2 (d), 54.7 (d), 25.6 (q); MS (FAB) $m / z 730\left[\mathrm{M}^{+}\right]$. Anal. Calcd. for $\mathrm{C}_{50} \mathrm{H}_{38} \mathrm{~N}_{2} \mathrm{O}_{4}$. THF : C, 80.77; H, 5.77; N, 3.49. Found: C, 80.67; H, $5.64 ; \mathrm{N}, 3.56$.

$\mathrm{N}$-(naphthylcarbonyl) \{4-[N-(naphthylcarbonyl)-N-(1S)-1-naphthylethyl]carbamoyl $\}$ naphthyl $\}-\mathrm{N}-((1 S)-$ 1-naphthylethyl)carboxamide 3c. Colorless crystals, m.p. 150-151 C. IR (KBr) 3049 (m), 1740 (m), 1702 (m), 1656 (s), 1509 (m) cm ${ }^{-1}$; UV-vis $\left(\mathrm{CH}_{3} \mathrm{CN}\right) 220$ (130000), 255 (25000), 356 (5500) nm; ${ }^{1} \mathrm{H}$ $\operatorname{NMR}\left(\mathrm{CDCl}_{3}, 500 \mathrm{MHz}\right) \delta 8.68(\mathrm{~d}, J=6.7,2 \mathrm{H}), 8.09(\mathrm{~d}, J=7.9,2 \mathrm{H}), 7.97(\mathrm{~d}, J=9.0,2 \mathrm{H}), 7.95(\mathrm{~d}, J=$ $7.6,2 \mathrm{H}), 7.83(\mathrm{t}, J=7.3,2 \mathrm{H}), 7.77(\mathrm{t}, J=7.0,2 \mathrm{H}), 7.63(\mathrm{t}, J=7.6,2 \mathrm{H}), 7.24(\mathrm{~m}, 4 \mathrm{H}), 6.98(\mathrm{br}, 4 \mathrm{H})$, $6.87(\mathrm{q}, J=6.6,2 \mathrm{H}), 6.75-6.70(\mathrm{~m}, 4 \mathrm{H}), 6.52(\mathrm{br}, 2 \mathrm{H}), 6.29(\mathrm{~m}, 4 \mathrm{H}), 5.87(\mathrm{br}, 2 \mathrm{H}), 2.21(\mathrm{~d}, J=6.7,6 \mathrm{H})$; ${ }^{13} \mathrm{C} \mathrm{NMR}\left(\mathrm{CDCl}_{3}, 125 \mathrm{MHz}\right) \delta 172.5$ (s), 135.5 (s), 134.4 (s), 134.1 (s), 133.8 (s), 131.91 (s), 131.87 (s), 129.6 (d), 129.3 (d), 128.7 (d), 128.0 (d), 127.5 (d), 127.3 (s), 126.6 (d), 126.44 (d), 126.39 (d), 126.1 
(d), 125.8 (d), 125.2 (d), 124.4 (d), 124.1 (d), 123.8 (d), 123.6 (d), 123.5 (d), 51.5 (d), 18.1 (q); MS (FAB) $m / z 830\left[\mathrm{M}^{+}\right]$. Anal. Calcd. for $\mathrm{C}_{58} \mathrm{H}_{42} \mathrm{~N}_{2} \mathrm{O}_{4} \cdot \mathrm{H}_{2} \mathrm{O}: \mathrm{C}, 82.05 ; \mathrm{H}, 5.22 ; \mathrm{N}, 3.30$. Found: C, 82.25; H, 5.32; N, 3.25.

$\mathrm{N}$-(naphthylcarbonyl) \{4-[N-(naphthylcarbonyl)-N-(2-tert-butyl-phenyl)carbamoyl]naphthyl\}-N-(2-tertbutyl-phenyl)carboxamide 3d. Colorless crystals, m.p. 238-240 ㄷ․ IR (KBr) 2964 (w), 1707 (m), 1670 (s), $1316(\mathrm{~m}) \mathrm{cm}^{-1}$; UV-vis $\left(\mathrm{CH}_{3} \mathrm{CN}\right) 258$ (20000), 304 (13000), 344 (7200) nm; ${ }^{1} \mathrm{H}$ NMR (CDCl, 500 MHz) $\delta 7.88$ (br, 2H), $7.73(\mathrm{~m}, 2 \mathrm{H}), 7.69$ (dd, $J=8.0,2.0,2 \mathrm{H}), 7.48-7.44(\mathrm{~m}, 6 \mathrm{H}), 7.39$ (m, 2H), 7.35 (d, $J=8.3,2 \mathrm{H}), 7.27(\mathrm{br}, 2 \mathrm{H}), 7.23(\mathrm{t}, J=7.3,2 \mathrm{H}), 7.10(\mathrm{br}, 2 \mathrm{H}), 7.02(\mathrm{~d}, J=8.3,2 \mathrm{H}), 6.60$ (br, 2H), 6.37 (br, 2H), 1.53 (s, 18H); ${ }^{13} \mathrm{C} \mathrm{NMR}\left(\mathrm{CDCl}_{3}, 125 \mathrm{MHz}\right) \delta 147.6$ (s), 137.4 (s), 134.6 (s), 133.1 (s), 132.6 (s), 131.1 (d), 130.3(s), 129.2(d), 129.1(d), 128.8 (d), 127.6 (d), 127.1 (d), 126.4 (d), 125.3 (d), 124.4 (d), 122.9 (d), 121.4 (d), 36.0 (s), 31.9 (q); MS (FAB) m/z 787 [MH']. Anal. Calcd. for $\mathrm{C}_{54} \mathrm{H}_{46} \mathrm{~N}_{2} \mathrm{O}_{4}$. $1 / 2 \mathrm{H}_{2} \mathrm{O}: \mathrm{C}, 81.48 ; \mathrm{H}, 5.95 ; \mathrm{N}, 3.52$. Found: C, 81.37; H, 5.84; N, 3.46 .

$\mathrm{N}$-(naphthylcarbonyl) $\{4-[\mathrm{N}-($ naphthylcarbonyl)-N-octylcarbamoyl]naphthyl $\}$-N-octylcarboxamide 3e. Colorless crystals, m.p. $95^{\circ} \mathrm{C}$. IR (KBr) 2925 (m), 2856 (m), 1700 (m), 1658 (s) cm ${ }^{-1}$; UV-vis $\left(\mathrm{CH}_{3} \mathrm{CN}\right)$ 255 (26000), 300 (14000), 345 (7900) nm; ${ }^{1} \mathrm{H} \mathrm{NMR}\left(\mathrm{CDCl}_{3}, 500 \mathrm{MHz}\right) \delta 7.67$ (d, $\left.J=8.2,2 \mathrm{H}\right), 7.46$ (br, $2 \mathrm{H}), 7.40(\mathrm{~d}, J=7.7,2 \mathrm{H}), 7.30(\mathrm{td}, J=7.5,1.3,2 \mathrm{H}), 7.25(\mathrm{td}, J=7.5,1.3,2 \mathrm{H}), 7.20(\mathrm{~m}, 2 \mathrm{H}), 7.10(\mathrm{~d}, J$ $=8.3,2 \mathrm{H}), 6.96(\mathrm{dd}, J=7.0,0.9,2 \mathrm{H}), 6.54(\mathrm{t}, J=7.5,2 \mathrm{H}), 6.44(\mathrm{~s}, 2 \mathrm{H}), 4.07(\mathrm{t}, J=7.2,4 \mathrm{H}), 1.82(5, J=$ 7.2, 4H), 1.52- $.23(\mathrm{~m}, 20 \mathrm{H}), 0.93(\mathrm{t}, J=6.9,6 \mathrm{H}) ;{ }^{13} \mathrm{C} \mathrm{NMR}\left(\mathrm{CDCl}_{3}, 125 \mathrm{MHz}\right) \delta 173.0(\mathrm{~s}), 171.7(\mathrm{~s})$, 135.6 (s), 133.9 (s), 132.6 (s), 130.3 (d), 128.9 (s), 128.50 (s), 128.45 (d), 127.0 (d, 3), 126.2 (d), 125.9 $(\mathrm{d}), 124.8(\mathrm{~d}), 124.3(\mathrm{~d}), 123.4(\mathrm{~d}), 123.0(\mathrm{~d}), 45.4(\mathrm{t}), 31.9(\mathrm{t}), 29.4(\mathrm{t}), 29.3(\mathrm{t}), 28.8(\mathrm{t}), 27.2(\mathrm{t}), 22.7(\mathrm{t})$, 14.2 (q); MS (FAB) $m / z 746\left[\mathrm{M}^{+}\right]$. Anal. Calcd. for $\mathrm{C}_{50} \mathrm{H}_{54} \mathrm{~N}_{2} \mathrm{O}_{4}: \mathrm{C}, 80.40 ; \mathrm{H}, 7.29 ; \mathrm{N}, 3.75$. Found: $\mathrm{C}$, $80.05 ; \mathrm{H}, 7.25 ; \mathrm{N}, 3.61$.

Compound 5a. Compound 2a (834mg, $1.52 \mathrm{mmol})$ and DMAP (187 mg, $1.53 \mathrm{mmol})$ in toluene (20 ml) were added to the toluene solution of 1,4-naphthalenedicarbonylchloride prepared from 1,4naphthalenedicarboxylic acid $(300 \mathrm{mg}, 1.39 \mathrm{mmol})$ with thionylchloride, and then the solution was refluxed for $20 \mathrm{~h}$. After cooling to room temperature, the solvent was evaporated and the residue was purified twice by silica gel column chromatography with $n$-hexane / ethylacetate $(2: 1)$ and chloroform / methanol (99:1) as eluent. Further purification by preparative HPLC (column: Merck Si 60) with nhexane / ethylacetate $(3: 1)$ as eluent gave 5a as a white powder $(61 \mathrm{mg}, 6.3 \%) ; \mathrm{mp} .156{ }^{\circ} \mathrm{C} . \mathrm{IR}(\mathrm{KBr})$ 2926 (w), 1703 (m), 1661 (s), 1316 (m) cm ${ }^{-1}$; UV-vis $\left(\mathrm{CHCl}_{3}\right) 311$ (13000), 370 (6500) nm; ${ }^{1} \mathrm{H}$ NMR $\left(\mathrm{CDCl}_{3}, 500 \mathrm{MHz}\right) \delta 7.57$ (d, $\left.J=7.0,4 \mathrm{H}\right), 7.45-7.31(\mathrm{~m}, 20 \mathrm{H}), 7.26(\mathrm{br}, 2 \mathrm{H}), 7.19-7.17(\mathrm{~m}, 2 \mathrm{H}), 7.02-$ $6.99(\mathrm{~m}, 6 \mathrm{H}), 6.90-6.88(\mathrm{~m}, 6 \mathrm{H}), 6.84(\mathrm{t}, J=7.4,2 \mathrm{H}), 6.71(\mathrm{~d}, J=6.7,4 \mathrm{H}), 6.35(\mathrm{t}, J=7.4,2 \mathrm{H}), 6.04$ 
(br, 2H), 5.91 (br, 2H), $5.82(\mathrm{br}, 2 \mathrm{H}), 5.20(\mathrm{~s}, 4 \mathrm{H}), 4.94(\mathrm{~s}, 4 \mathrm{H}) ;{ }^{13} \mathrm{C} \mathrm{NMR}\left(\mathrm{CDCl}_{3}, 125 \mathrm{MHz}\right) \delta 172.7$ (s), 170.9(s), 170.8 (s), 137.3 (s), 137.0 (s), 135.4 (s), 134.9 (s), 134.7 (s), 133.4 (s), 132.2 (s), 130.0 (d), 129.4 (d), 129.3 (d), 128.57 (d), 128.55 (d), 128.5 (d), 128.0 (d), 127.9 (d), 127.80 (d), 127.77 (d), 127.7 (d), 127.6 (d), 126.91 (d), 126.85 (d), 126.8 (d), 126.1 (d), 125.7 (d), 124.9 (d), $124.3(\mathrm{~d}), 123.9$ (d), 123.7 (d), 123.3 (d), 122.9 (d), 76.8 (d), 48.2 (t), 48.1 (t); MS (FAB) m/z 1277 [MH ${ }^{+}$. Anal. Calcd. for $\mathrm{C}_{86} \mathrm{H}_{60} \mathrm{~N}_{4} \mathrm{O}_{8} \cdot 2 \mathrm{H}_{2} \mathrm{O}: \mathrm{C}, 78.64 ; \mathrm{H}, 4.91 ; \mathrm{N}, 4.27$. Found: C, 78.45; H, 4.54; N, 4.01.

In a similar manner as for $\mathbf{5 a}, \mathbf{5 b}$ was prepared in $9.9 \%$ yield. Compound $\mathbf{5 b}$. White powder, mp. $225{ }^{\circ} \mathrm{C}$. IR (KBr) 2930 (w), 1704 (s), 1655 (s), 1508 (m) cm ${ }^{-1}$; UV-vis $\left(\mathrm{CH}_{3} \mathrm{CN}\right) 213$ (140000), 259 (29000), 369 (5400) nm; ${ }^{1} \mathrm{H} \mathrm{NMR}\left(\mathrm{CDCl}_{3}, 400 \mathrm{MHz}\right) \delta 7.92$ (d, $\left.J=8.0,2 \mathrm{H}\right), 7.75$ (d, $\left.J=7.6,2 \mathrm{H}\right), 7.54$ $(\mathrm{d}, J=8.5,2 \mathrm{H}), 7.43-7.06(\mathrm{~m}, 22 \mathrm{H}), 6.94-6.80(\mathrm{~m}, 10 \mathrm{H}), 6.66(\mathrm{~d}, J=5.0,2 \mathrm{H}), 6.50(\mathrm{t}, J=8.0,2 \mathrm{H})$, $6.32(\mathrm{~d}, J=6.6,2 \mathrm{H}), 6.05(\mathrm{~m}, 2 \mathrm{H}), 5.58-5.54(\mathrm{~m}, 4 \mathrm{H}), 5.44(\mathrm{~s}, 2 \mathrm{H}), 5.32(\mathrm{q}, J=7.3,2 \mathrm{H}), 5.05(\mathrm{t}, J=7.6$, $2 \mathrm{H}), 1.80(\mathrm{~d}, J=7.6,6 \mathrm{H}), 1.78(\mathrm{~d}, J=7.3,6 \mathrm{H}) ;{ }^{13} \mathrm{C} \mathrm{NMR}\left(\mathrm{CDCl}_{3}, 100 \mathrm{MHz}\right) \delta 178.6,172.8,170.8$, 167.0, 143.9, 139.6, 134.4, 134.0, 132.6, 129.8, 129.6, 128.9, 128.8, 128.7, 128.61, 128.57, 128.5, 128.4, 128.2, 128.1, 127.6, 127.6, 127.4, 127.3, 127.2., 127.0, 126.4, 126.0, 125.9, 125.6, 125.1, 123.6, 123.5, 122.9, 43.1, 42.6, 16.5, 16.4; HRMS (FAB), calcd for $\mathrm{C}_{90} \mathrm{H}_{68} \mathrm{~N}_{4} \mathrm{O}_{8}\left(\mathrm{MH}^{+}\right)$1333.5115, found 1333.4996.

Compound 4c. Compound 2c (1.24 mg, $2.37 \mathrm{mmol})$ and DMAP (583 mg, $4.77 \mathrm{mmol})$ in toluene (45 ml) and pyridine $(7 \mathrm{ml})$ was added to the toluene solution of 1,4-naphthalenedicarbonylchloride prepared from 1,4-naphthalenedicarboxylic acid (500 mg, $2.31 \mathrm{mmol})$ with thionylchloride, and then the resulting mixture was refluxed for 3 day. After cooling to room temperature, the solution was washed with aqueous $1 \mathrm{~N} \mathrm{HCl}$ solution and brine. The organic layer separated was dried over $\mathrm{MgSO}_{4}$, filtered, and evaporated. The residue was purified by silica gel column chromatography with $n$-hexane / ethylacetate $(2: 1)$ as eluent to give $4 \mathrm{c}(527 \mathrm{mg}, 36 \%) ;{ }^{1} \mathrm{H} \mathrm{NMR}\left(\mathrm{CDCl}_{3}, 400 \mathrm{MHz}\right) \delta 8.09(\mathrm{~d}, J=8.8,2 \mathrm{H}), 8.01$ $7.43(\mathrm{~m}, 32 \mathrm{H}), 7.07-6.57(\mathrm{~m}, 12 \mathrm{H}), 6.13$ (br, 2H), 5.99 (quint, $J=6.9,2 \mathrm{H}), 4.86$ (br, 2H), 2.32 (d, $J=$ $5.1,6 \mathrm{H}), 1.83(\mathrm{~d}, J=6.5,6 \mathrm{H}) ;{ }^{13} \mathrm{C} \mathrm{NMR}\left(\mathrm{CDCl}_{3}, 125 \mathrm{MHz}\right) \delta 166.1,134.6,134.1,133.8,133.6,131.9$, $131.5,129.5,128.9,128.6,128.4,127.9,126.8,126.7,126.6,126.5,125.9,125.8,125.1,125.0,124.0$, $123.5,123.0,121.8,60.4,51.3,44.2,21.1,19.7,17.8,14.2$; MS (FAB) $m / z 1225\left[\mathrm{MH}^{+}\right]$.

Compound 5c. Compound 4c (299 mg, $0.244 \mathrm{mmol})$ and DMAP (119 mg, $0.976 \mathrm{mmol})$ in $20 \mathrm{ml}$ of dry toluene was refluxed for $3 \mathrm{~h}$ with molecular sieves and then naphthalene-1-carbonylchloride $(0.15 \mathrm{ml}$, $0.996 \mathrm{mmol}$ ) was added to the solution. The resulting mixture was refluxed for $48 \mathrm{~h}$. After cooling to room temperature, the solution was washed with diluted $\mathrm{HCl}$ and brine. The organic layer was dried over $\mathrm{MgSO}_{4}$. After filtration, the solvent was evaporated and the residue was purified by silica gel column chromatography with $n$-hexane / ethylacetate $(3: 1)$ as eluent. Further purification by 
preparative HPLC (column; Merck Si 60) eluted with $n$-hexane / ethylacetate $(3: 1)$ gave 5c as a white powder (54 mg, 14 \%); m.p. 187-190 ${ }^{\circ} \mathrm{C}$. IR (KBr) $3058(\mathrm{w}), 1702(\mathrm{~m}), 1656(\mathrm{~s}), 1510$ (m) cm ${ }^{-1}$; UV-vis $\left(\mathrm{CH}_{3} \mathrm{CN}\right) 273$ (43000), 295 (29000), 375 (10000) nm; ${ }^{1} \mathrm{H} \mathrm{NMR}\left(\mathrm{CDCl}_{3}, 500 \mathrm{MHz}\right) \delta 8.59$ (d, J=8.0, $2 \mathrm{H}), 8.37(\mathrm{~d}, J=8.0,2 \mathrm{H}), 8.07(\mathrm{~d}, J=8.0,2 \mathrm{H}), 8.02-7.92(\mathrm{~m}, 8 \mathrm{H}), 7.83(\mathrm{~d}, J=7.0,2 \mathrm{H}), 7.75-7.60(\mathrm{~m}$, $14 \mathrm{H}), 7.17-6.68(\mathrm{~m}, 10 \mathrm{H}), 6.81(\mathrm{q}, J=6.7,2 \mathrm{H}), 6.59-6.52(\mathrm{~m}, 10 \mathrm{H}), 6.53(\mathrm{q}, J=6.4,2 \mathrm{H}), 6.09(\mathrm{br}, 2 \mathrm{H})$, $5.80(\mathrm{br}, 2 \mathrm{H}), 5.64-5.51(\mathrm{~m}, 6 \mathrm{H}), 2.15(\mathrm{~d}, J=6.7,6 \mathrm{H}), 1.95(\mathrm{~d}, J=6.4,6 \mathrm{H}) ;{ }^{13} \mathrm{C} \mathrm{NMR}\left(\mathrm{CDCl}_{3}, 125\right.$ MHz) $\delta 172.3$ (s), 170.6 (s), 170.3 (s), 169.8 (s), 135.6 (s), 135.4 (s), 135.1 (s), 134.03 (s), 134.00 (s), 133.9 (s), 133.8 (s), 133.6 (s), 131.8 (s), 129.5 (d), 129.2 (d), 128.57 (d), 128.55 (d), 128.0 (d), 127.8 (d), 127.6 (d), 127.0 (s), 126.6 (d), 126.5, (d) 126.4 (d), 126.3 (s), 126.3 (d), 126.1 (s), 125.9 (s), 125.83 (d), 125.79 (d), 125.14 (d), 125.06 (d), 124.5 (d), 123.9 (d), 123.5 (d), 123.41 (d), 123.35 (d), 51.3 (d), 51.1 (d), 18.0 (q), 17.8 (q); HRMS (FAB), calcd for $\mathrm{C}_{106} \mathrm{H}_{76} \mathrm{~N}_{4} \mathrm{O}_{8}\left(\mathrm{MH}^{+}\right)$1533.5741, found 1533.5690. 


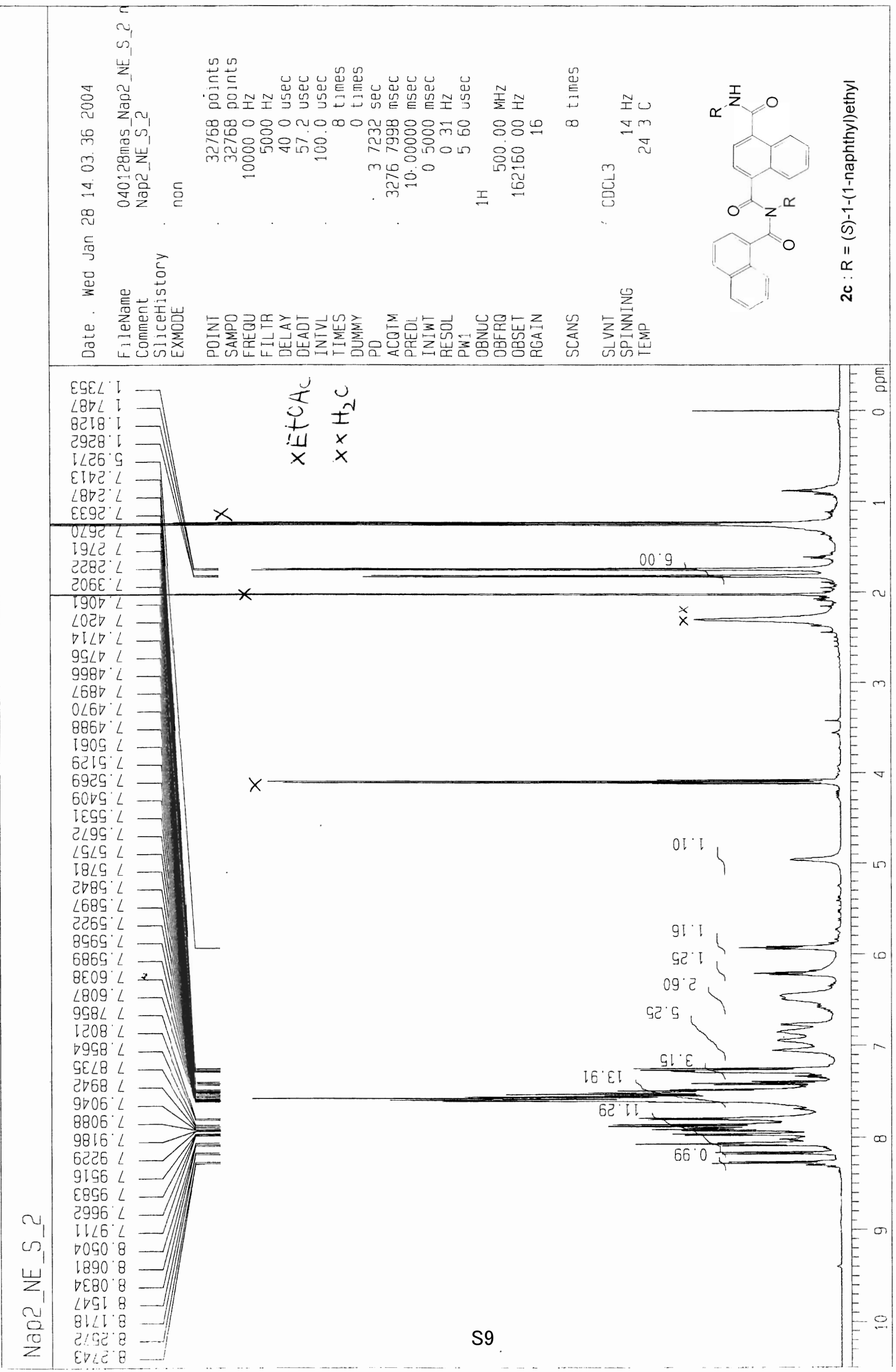




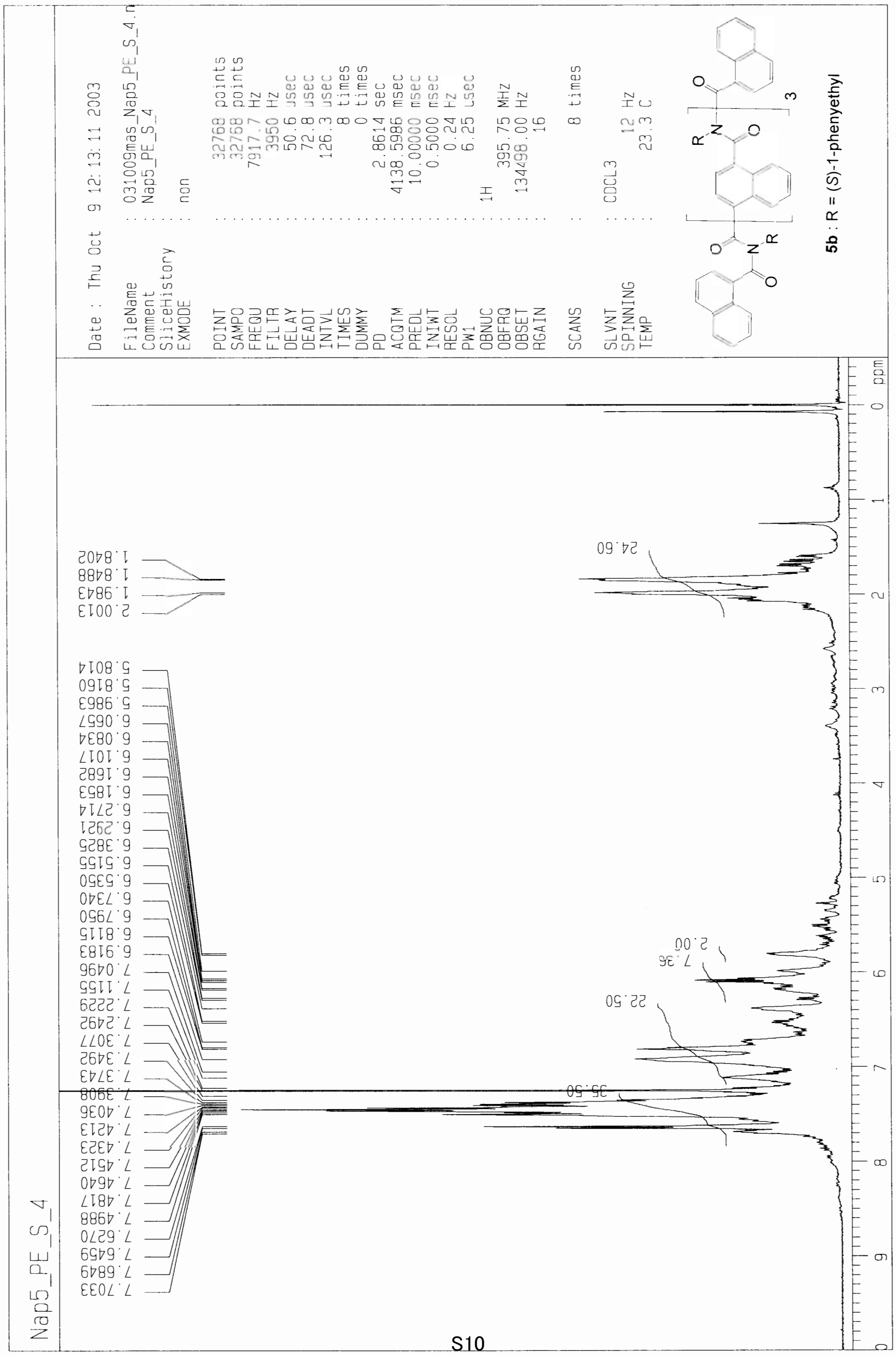




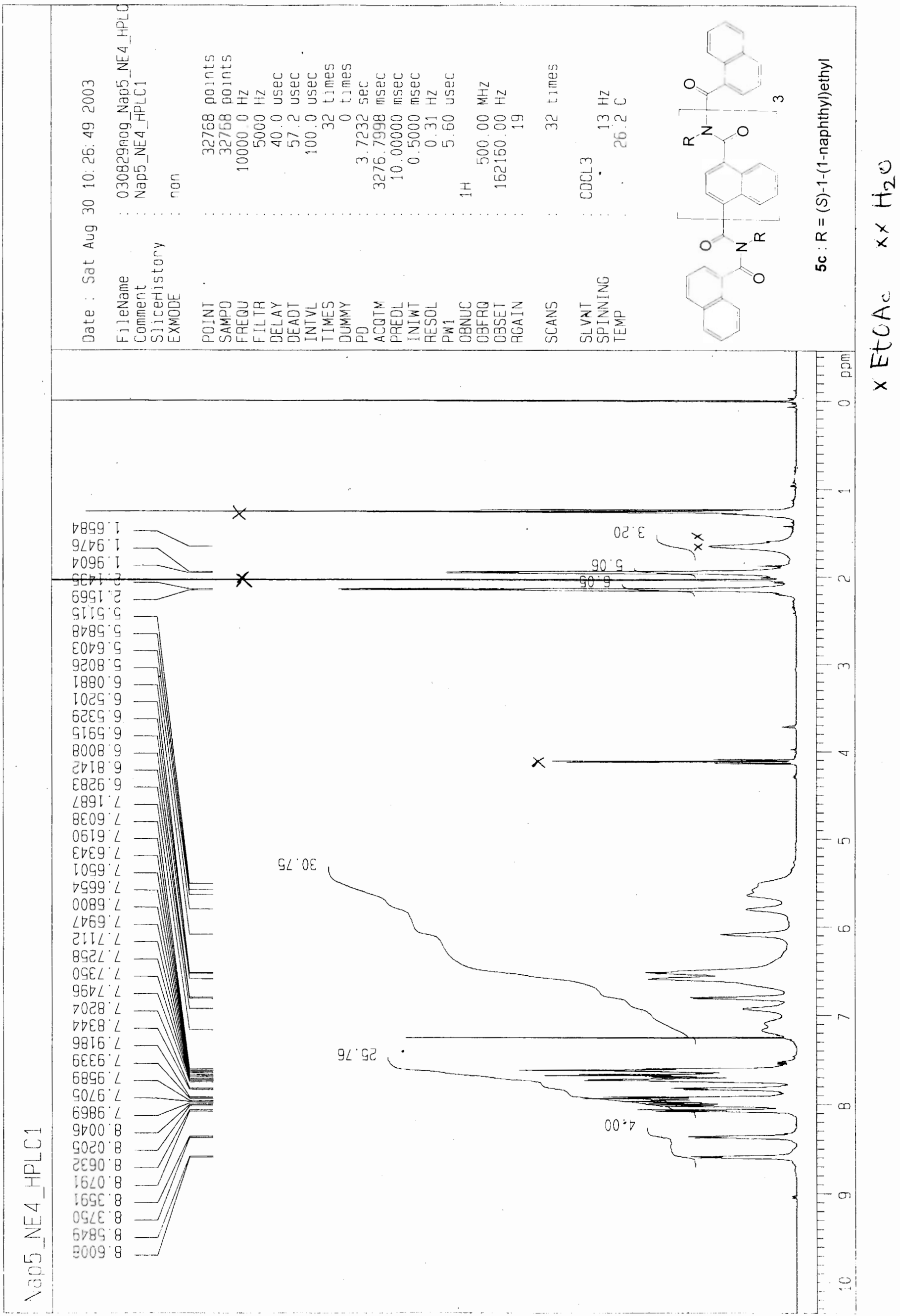




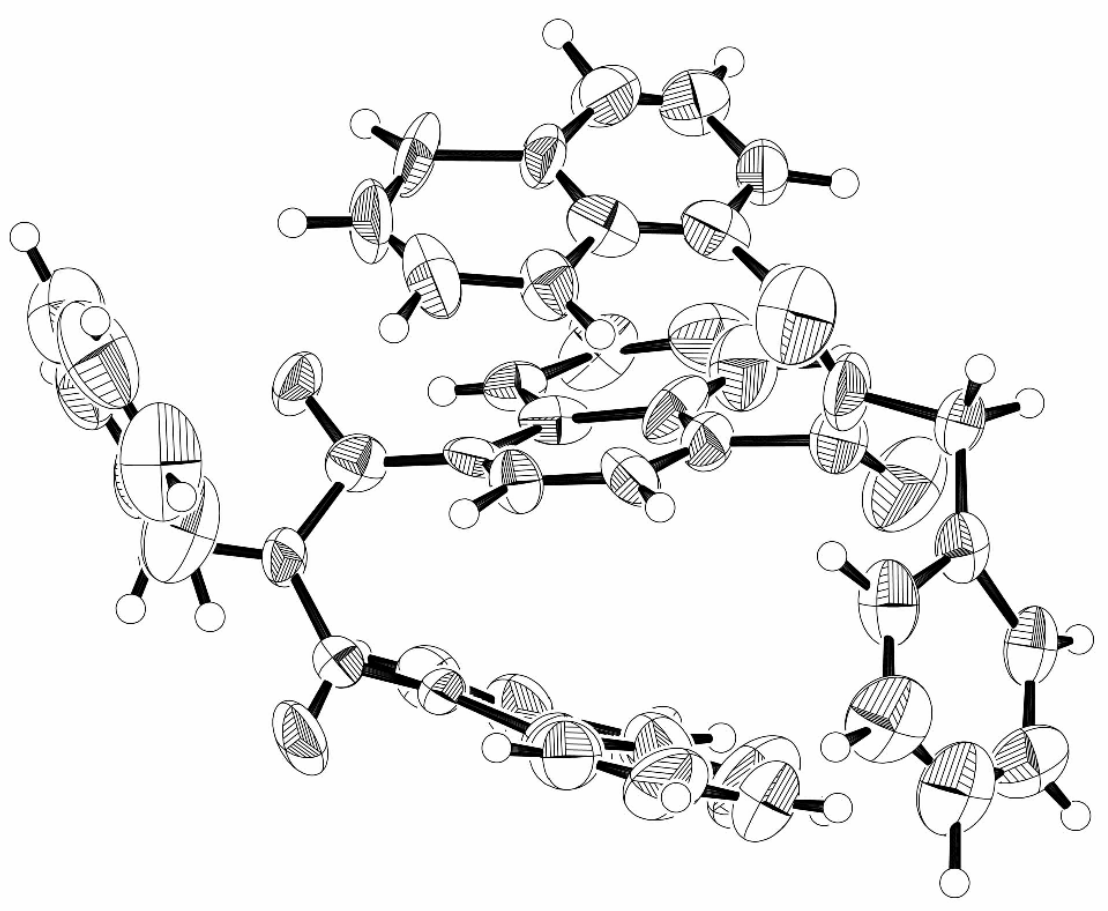

ORTEP diagram of $\mathbf{3 a}$ 

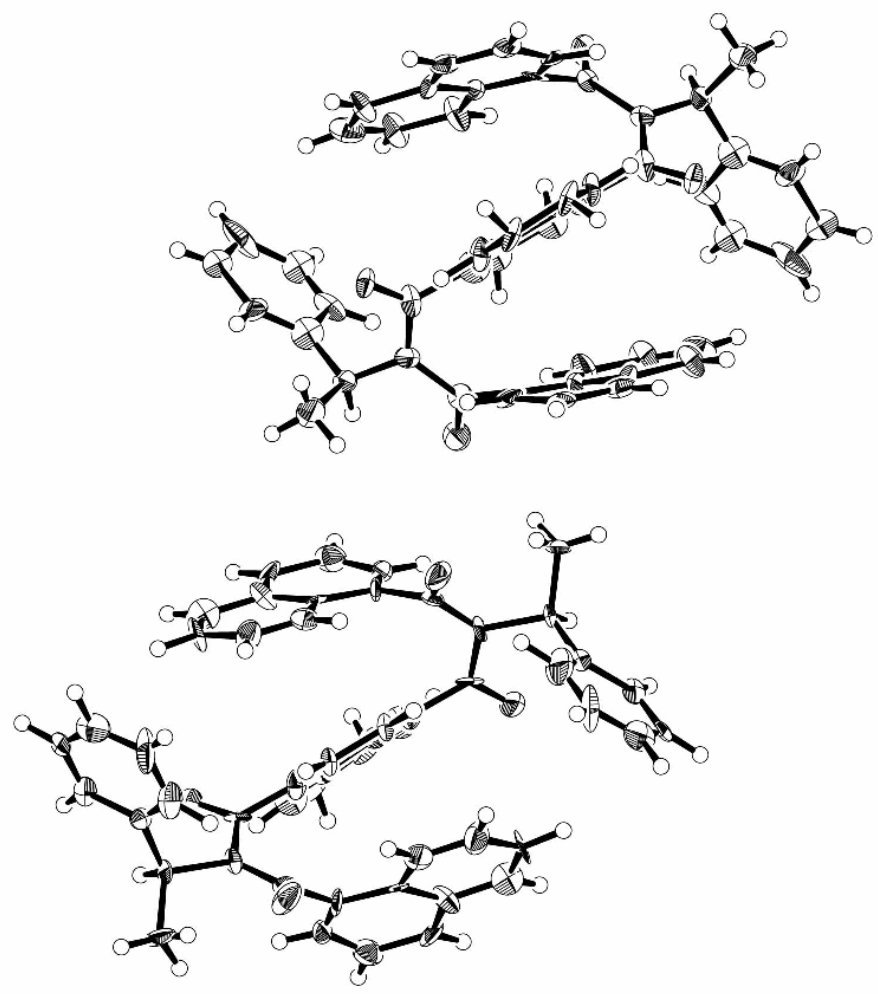

ORTEP diagram of $\mathbf{3 b}$ 


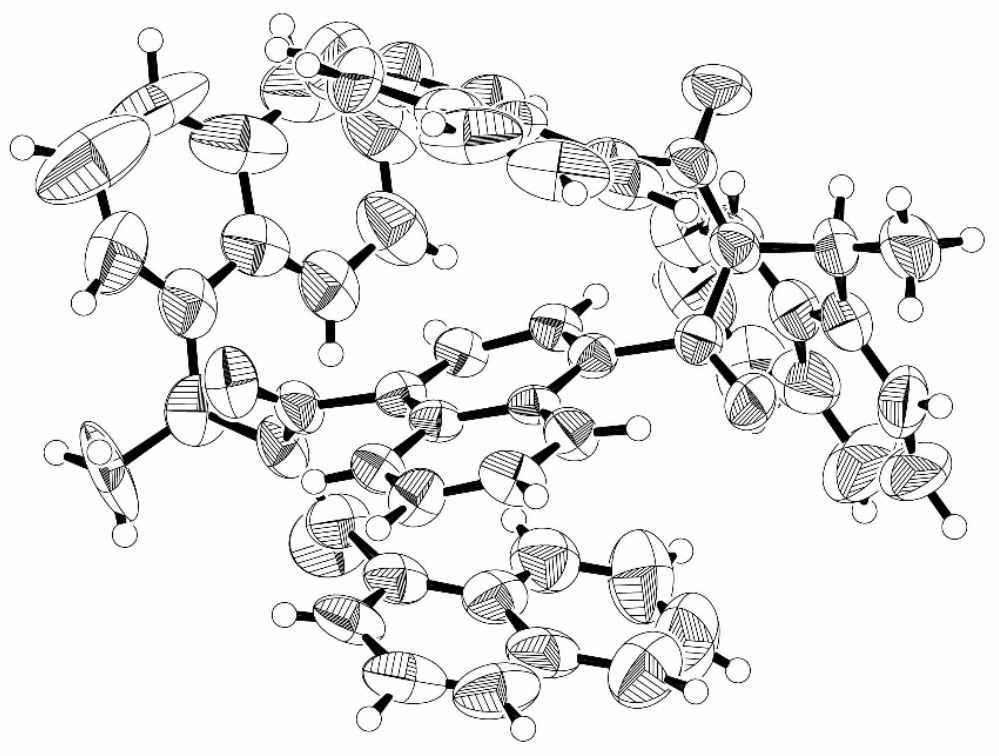

ORTEP diagram of $\mathbf{3 c}$ 


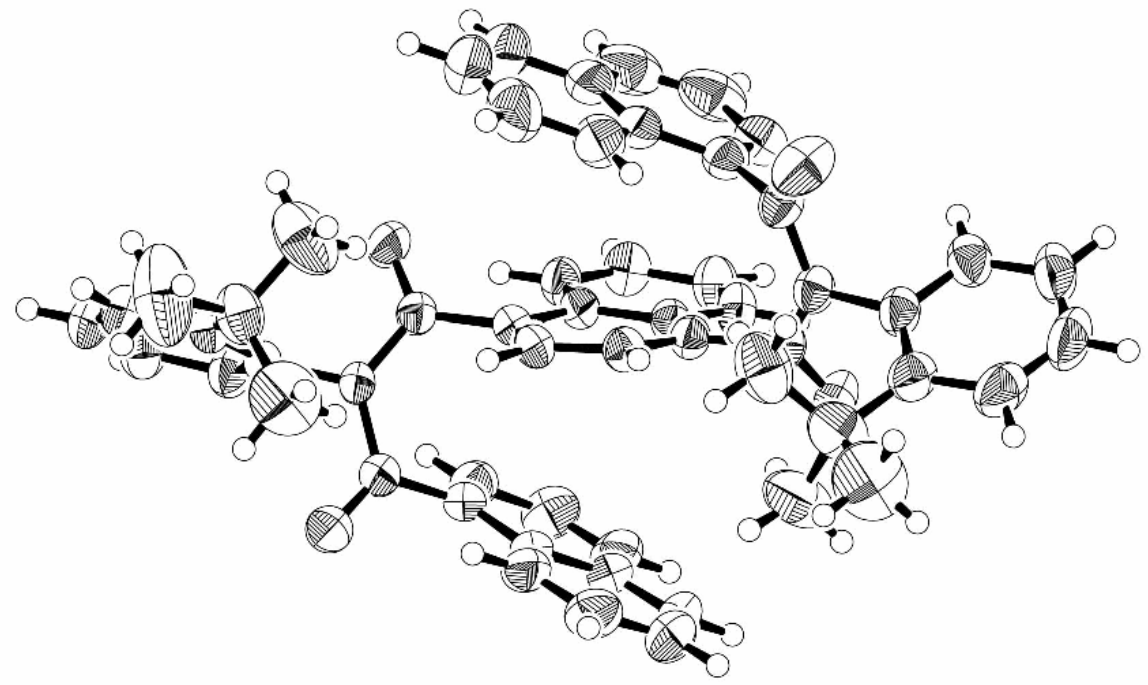

ORTEP diagram of $\mathbf{3 d}$ 


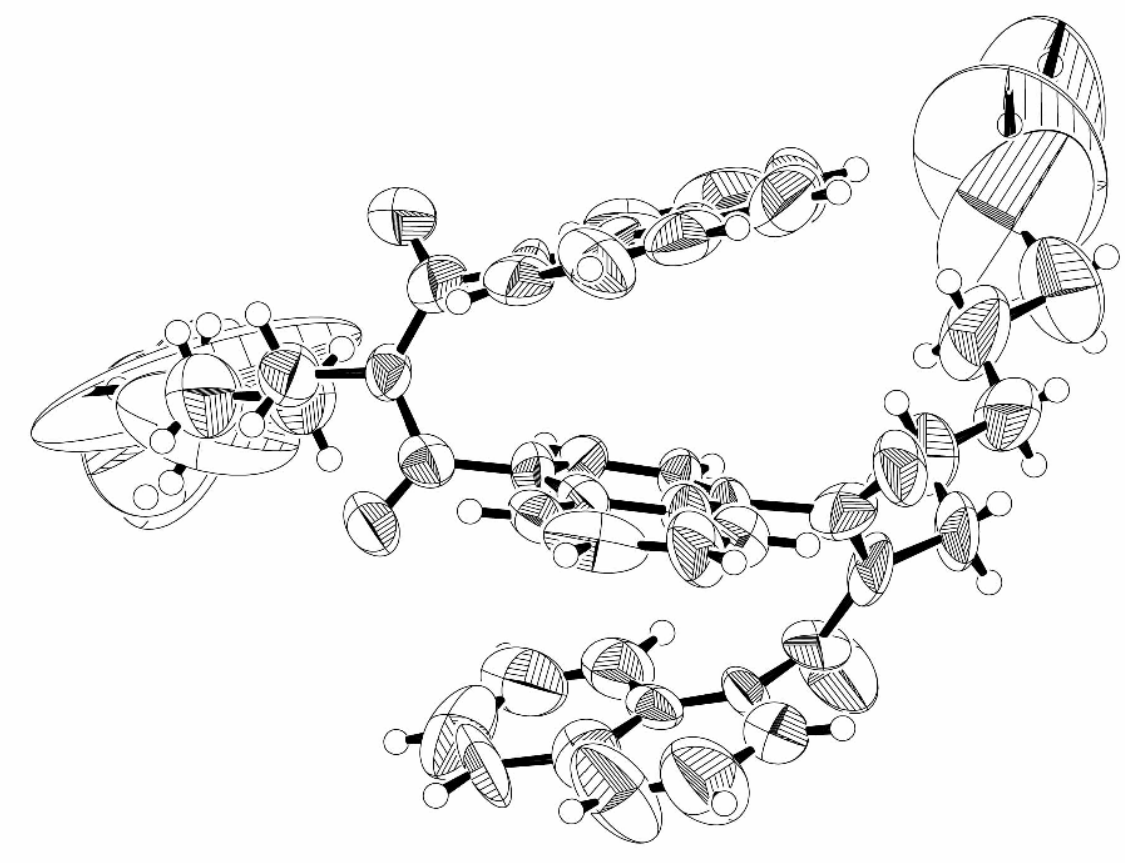

ORTEP diagram of $\mathbf{3 e}$ 